\section{Stability Analysis and Stabilization of Systems Presenting Nested Saturations}

S. Tarbouriech, C. Prieur, and J. M. Gomes da Silva, Jr.

\begin{abstract}
This note addresses the problems of stability analysis and stabilization of systems presenting nested saturations. Depending on the open-loop stability assumption, the global stability analysis and stabilization problems are considered. In the (local) analysis problem, the objective is the determination of estimates of the basin of attraction of the system. Considering the stabilization problem, the goal is to design a set of gains in order to enlarge the basin of attraction of the closed-loop system. Based on the modelling of the system presenting nested saturations as a linear system with dead-zone nested nonlinearities and the use of a generalized sector condition, linear matrix inequality (LMI) stability conditions are formulated. From these conditions, convex optimization strategies are proposed to solve both problems.
\end{abstract}

Index Terms-Linear matrix inequality (LMI), nested saturations, stability regions, stabilization.

\section{INTRODUCTION}

The stability and stabilization of systems presenting saturations are problems that have been studied by many authors with different objectives in the last decade. This interest comes mainly from the fact that, due to physical, safety or technological constraints, control actuators cannot provide unlimited amplitude signals neither unlimited speed of reaction. This means that control systems are in general subject to amplitude and dynamics actuator saturations. In this case, the negligence of both amplitude and dynamics actuator limitations in control systems can be source of undesirable and even catastrophic behaviors, such as instability [3].

In this context, an important class of systems to be studied consists in systems presenting nested saturations. In particular, such structure appears when we deal with nonlinear actuators and sensors. For instance, it is common in aerospace control systems (e.g., launcher and aircraft control) that actuators are both limited in amplitude and rate (dynamics) (see, for example, [1], [8], and [10]). Furthermore, the presence of both sensor and actuator amplitude limitations can lead to a closed-loop system presenting nested saturations. This will be the case, for instance, in linear systems controlled by dynamic output feedback controllers in the presence saturating sensors and actuators. On the other hand, analysis and design methodologies for systems presenting nested saturations can be useful to address stability issues of more general classes of nonlinear systems. For instance, the use of nested saturations becomes very interesting when one uses forwarding techniques for cascade systems with linear part [15], [17], [18].

In this note, two complementary problems are addressed: The determination of stability regions and the design of stabilizing gains for systems presenting nested saturations. The objective in the analysis stability problem consists in determining an estimate of the basin of attraction of the system presenting generic nested saturations. Regarding the

Manuscript received June 2, 2004; revised October 10, 2005 and April 11, 2006. Recommended by Associate Editor M. Kothare. The work of J. M. Gomes da Silva, Jr. was supported by CNPq, Brazil.

S. Tarbouriech and C. Prieur are with LAAS-CNRS, 31077 Toulouse cedex 4, France (e-mail: tarbour,cprieur@laas.fr).

J. M. Gomes da Silva Jr. is with the Department of Electrical Engineering, UFRGS, 90035-190 Porto Alegre-RS, Brazil (e-mail: jmgomes@ece.ufrgs.br). Digital Object Identifier 10.1109/TAC.2006.878743 stabilization problem, the objective is then to design a set of gains in order to maximize the basin of attraction of the closed-loop saturated system. Based on the modelling of the system presenting generic nested saturations as a linear system with dead-zone nested nonlinearities and the use of a generalized sector condition, linear matrix inequality (LMI) stability conditions are formulated. Although the objectives of the note are similar to the ones recently proposed in [1], considering the modelling of the saturated systems by a polytopic differential inclusion, it should be pointed out that we do not consider particular assumptions on the structure and the dimensions of matrices involved in the description of the system. Furthermore, our results allow also to address global stability issues, which is not considered in [1]. This note provides therefore an alternative solution for the problems formulated and addressed in [1] and, doing so, provides also some generalizations.

The note is organized as follows. Section II describes the system under consideration and the problems we intend to address. In Section III, theoretical conditions for solving both the analysis of stability as well as the control design problems are presented. Section IV deals with convex optimization issues. A discussion concerning the numerical complexity with respect to our results and those proposed in the literature is quickly presented in the end of this section. Some illustrative examples are presented in Section V. Section VI summarizes the main contributions of the work and points to some open problems and future research directions.

Notations: For any vector $x \in \Re^{n}, x \succeq 0$ means that all the components of $x$, denoted $x_{(i)}$, are nonnegative. For two vectors $x, y$ of $\Re^{n}$, the notation $x \succeq y$ means that $x_{(i)}-y_{(i)} \geq 0, \forall i=1, \ldots, n$. 1 and 0 denote, respectively, the identity matrix and the null matrix of appropriate dimensions. The elements of a matrix $A \in \Re^{m \times n}$ are denoted by $A_{(i, j)}, i=1, \ldots, m, j=1, \ldots, n$. $A_{(i)}$ denotes the $i$ th row of matrix $A .|A|$ is the matrix constituted from the absolute value of each element of $A$. For two symmetric matrices, $A$ and $B, A>B$ means that $A-B$ is positive definite. $A^{\prime}$ denotes the transpose of $A$. Co denotes a convex hull.

\section{PRoblem Statement}

This note focuses on the following class of nonlinear systems resulting from nested saturations:

$$
\begin{aligned}
\dot{x}=A_{p} x+B_{p} \operatorname{sat}_{p}\left(A_{p-1} x+\right. & B_{p-1} \operatorname{sat}_{p-1}\left(A_{p-2} x\right. \\
& \left.+\cdots\left(A_{1} x+B_{1} \operatorname{sat}_{1}(C x)\right) \cdots\right)
\end{aligned}
$$

where $x \in \Re^{n}$ is the state of the system. For all $j \in\{1, \ldots, p\}, A_{j}$, $B_{j}$, and $C$ are matrices of appropriate dimensions (eventually having different dimensions depending on the index $j$ ). Furthermore, $s a t_{j}$ is a componentwise saturation map $\Re^{m_{j}} \rightarrow \Re^{m_{j}}$ defined as follows:

$$
\begin{aligned}
\left(\operatorname{sat}_{j}(v)\right)_{(i)}=\operatorname{sat}_{j}\left(v_{(i)}\right)=\operatorname{sign}\left(v_{(i)}\right) \min \left(u_{j(i)},\right. & \left.\left|v_{(i)}\right|\right), \\
& \forall i=1, \ldots, m_{j}
\end{aligned}
$$

where $u_{j(i)}$, denotes the $i$ th bound of the $j$ th saturation function. In contrast with [1], accordingly to the different $m_{j}$, more generic matrices $B_{j}, A_{j}$ and $C$ can be considered. In particular, note also that the matrices $B_{j}$ do not need to be diagonal.

For $j=1, \ldots p$, let us recursively define the nonlinearities as shown in (2) at the bottom of the next page. 
Define now the following $p$ matrices:

$$
\begin{aligned}
& \mathrm{A}_{1}=A_{1}+B_{1} C \\
& \mathrm{~A}_{2}=A_{2}+B_{2}\left(A_{1}+B_{1} C\right) \\
& \mathrm{A}_{p}=A_{p}+B_{p}\left(A_{p-1}+B_{p-1}\right. \\
& \left.\times\left(A_{p-2}+\cdots+B_{2}\left(A_{1}+B_{1} C\right)\right)\right) .
\end{aligned}
$$

Thus, from the definitions in (2) and (3), system (1) can be rewritten as

$$
\begin{aligned}
\dot{x}=\mathrm{A}_{p} x+B_{p} \phi_{p}(x)+B_{p} B_{p-1} \phi_{p-1}(x) & +\cdots \\
& +B_{p} B_{p-1} \cdots B_{1} \phi_{1}(x)
\end{aligned}
$$

Note that in the absence of saturation one gets $\phi_{j}(x)=\mathbf{0}$, $j=1, \ldots, p$. In this case, the stability of system (1) (or, equivalently, (4)) is directly characterized by the stability of the matrix $A_{p}$. In the presence of saturation, considering that the exact analytical determination of the basin of attraction of the system is in general not possible, we should be concerned with the determination of estimates of this basin, i.e., regions in the state space in which the asymptotic stability of system (1) is guaranteed.

Considering system (1) and the definitions in (2) and (3), the two problems we intend to solve can therefore be summarized as follows.

Problem 1: Given a system where the matrix $A_{p}$ is Hurwitz, determine a region of stability $\mathcal{E}_{0}$ for system (1) as large as possible. Problem 2: Determine the gains $A_{j}, j=1, \ldots, p-1$ and $C$ such that $A_{p}$ is Hurwitz and that lead to a region of stability $\mathcal{E}_{0}$ for system (1) as large as possible.

The two problems above are complementary. The first one consists in a stability analysis problem. The idea is to determine an estimate, as large as possible, of the basin of attraction of the nested saturated system (1). The second one is a synthesis problem. In this case, the implicit goal is to design the gains $A_{j}, j=1, \ldots, p-1$ and $C$ in order to maximize the basin of attraction of system (1). In order to develop our results concerning these two problems, in the sequel we will use the equivalent description (4) of system (1).

\section{THEORETICAL CONDITIONS}

\section{A. Local Asymptotic Stability Analysis}

Let us consider the generic nonlinearity $\varphi(v)=\operatorname{sat}_{v_{0}}(v)-v$, with $\varphi(v) \in \Re^{m}$ and $v_{0(i)}, i=1, \ldots, m$ denoting the bounds of the saturation function $s a t_{v_{0}}(v)$, and define the following associated set:

$$
S\left(v_{0}\right)=\left\{v \in \Re^{m} ; w \in \Re^{m} ;-v_{0} \preceq v-w \preceq v_{0}\right\} .
$$

Lemma 1: If $v$ and $w$ are elements of $S\left(v_{0}\right)$, then the nonlinearity $\varphi(v)$ satisfies the following inequality:

$$
\varphi(v)^{\prime} T(\varphi(v)+w) \leq 0
$$

for any diagonal positive-definite matrix $T \in \Re^{m \times m}$.

Proof: Assume that $v$ and $w$ are elements of $S\left(v_{0}\right)$. In this case, it follows that $v_{0(i)}-v_{(i)}+w_{(i)} \geq 0$ and $-v_{0(i)}-v_{(i)}+w_{(i)} \leq 0$. Consider now the following three cases.

- Case 1: $v_{(i)}>v_{0(i)}$ It follows that $\varphi\left(v_{(i)}\right)=v_{0(i)}-v_{(i)}<0$ and one gets $\varphi\left(v_{(i)}\right) T_{(i, i)}\left(\varphi\left(v_{(i)}\right)+w_{(i)}\right)=\varphi\left(v_{(i)}\right) T_{(i, i)}\left(v_{0(i)}-\right.$ $\left.v_{(i)}+w_{(i)}\right) \leq 0$ provided that $T_{(i, i)}>0$.

- Case 2: $-v_{0(i)} \leq v_{(i)} \leq v_{0(i)}$ It follows that $\varphi\left(v_{(i)}\right)=0$ and $\varphi\left(v_{(i)}\right) T_{(i, i)}\left(\varphi\left(v_{(i)}\right)+w_{(i)}\right)=0, \forall T_{(i, i)}$.

- Case 3: $v_{(i)}<-v_{0(i)}$ It follows that $\varphi\left(v_{(i)}\right)=-v_{0(i)}-v_{(i)}>0$ and $\varphi\left(v_{(i)}\right) T_{(i, i)}\left(\varphi\left(v_{(i)}\right)+w_{(i)}\right)=\varphi\left(v_{(i)}\right) T_{(i, i)}\left(-v_{0(i)}-v_{(i)}+\right.$ $\left.w_{(i)}\right) \leq 0$ provided that $T_{(i, i)}>0$

Thus, once $v$ and $w$ are elements of $S\left(v_{0}\right)$, we can conclude that $\varphi\left(v_{(i)}\right) T_{(i, i)}\left(\varphi\left(v_{(i)}\right)+w_{(i)}\right) \leq 0, \forall T_{(i, i)}>0, \forall i=1, \ldots, m$, whence follows (6).

Remark 1: Particular formulations of Lemma 1 can be found in [7] (concerning the case of systems with a single saturation function, i.e., $p=1$ ) and in [16] (concerning systems presenting both amplitude and dynamics restricted actuators).

It should be pointed out that sector condition (6) is more generic than the classical one (see, for instance, [9] and [11]) given as

$$
\psi(v)^{\prime} T[\psi(v)+\lambda v] \leq 0, \quad 0<\lambda \leq 1 .
$$

Note that in our case we can consider $w \neq \lambda v$. This fact, differently from condition (7), allows to formulate conditions directly in LMI form.

The following proposition provides theoretical sufficient conditions to solve Problem 1.

Proposition 1: If there exist a symmetric positive definite matrix $W$, matrices $Z_{j j}, j=1, \ldots, p, Y_{j l}, j=2, \ldots, p, l=1, \ldots, p-1, j \neq l$,

$$
\begin{aligned}
\phi_{1}(x)= & \operatorname{sat}_{1}(C x)-C x \\
\phi_{2}(x)= & \operatorname{sat}_{2}\left(\left(A_{1}+B_{1} C\right) x+B_{1} \phi_{1}(x)\right)-\left[\left(A_{1}+B_{1} C\right) x+B_{1} \phi_{1}(x)\right] \\
\phi_{3}(x)= & \operatorname{sat}_{3}\left(\left(A_{2}+B_{2}\left(A_{1}+B_{1} C\right)\right) x+B_{2} \phi_{2}(x)+B_{2} B_{1} \phi_{1}(x)\right) \\
& \quad-\left[\left(A_{2}+B_{2}\left(A_{1}+B_{1} C\right)\right) x+B_{2} \phi_{2}(x)+B_{2} B_{1} \phi_{1}(x)\right] \\
& \vdots \\
\phi_{p}(x)= & \operatorname{sat}_{p}\left(\left(A_{p-1}+B_{p-1}\left(A_{p-2}+B_{p-2}\left(A_{p-3}+\cdots+B_{2}\left(A_{1}+B_{1} C\right)\right)\right)\right) x\right. \\
& \left.\quad+B_{p-1} \phi_{p-1}(x)+B_{p-1} B_{p-2} \phi_{p-2}(x)+\cdots+B_{p-1} B_{p-2} \cdots B_{1} \phi_{1}(x)\right) \\
& \quad-\left[\left(A_{p-1}+B_{p-1}\left(A_{p-2}+B_{p-2}\left(A_{p-3}+\cdots+B_{2}\left(A_{1}+B_{1} C\right)\right)\right)\right) x\right. \\
& \left.\quad+B_{p-1} \phi_{p-1}(x)+B_{p-1} B_{p-2} \phi_{p-2}(x)+\cdots+B_{p-1} B_{p-2} \cdots B_{1} \phi_{1}(x)\right]
\end{aligned}
$$


$j>l$, and diagonal positive matrices $S_{j}, j=1, \ldots, p$, of appropriate dimensions satisfying 1

$$
\begin{aligned}
& {\left[\begin{array}{ccccc}
W A_{p}^{\prime}+A_{p} W & \star & \star & \cdots & \star \\
S_{1} B_{1}^{\prime} \cdots B_{p-1}^{\prime} B_{p}^{\prime}-Z_{11} & -2 S_{1} & \star & \cdots & \star \\
S_{2}^{\prime} B_{2}^{\prime} \cdots B_{p-1}^{\prime} B_{p}^{\prime}-Z_{22} & -Y_{21} & -2 S_{2} & \cdots & \star \\
\vdots & \vdots & \vdots & \cdots & \star \\
S_{p}^{\prime} B_{p}^{\prime}-Z_{p p} & -Y_{p 1} & -Y_{p 2} & \cdots & -2 S_{p}
\end{array}\right]<0} \\
& {\left[\begin{array}{cc}
W & W C_{(i)}^{\prime}-Z_{11(i)}^{\prime} \\
\star & u_{1(i)}^{2}
\end{array}\right] \geq 0, \quad i=1, \ldots, m_{1}}
\end{aligned}
$$

and (10), as shown at the bottom of the page. Then, the set $\mathcal{E}\left(W^{-1}, 1\right)=\left\{x \in \Re^{n} ; x^{\prime} W^{-1} x \leq 1\right\}$ is a region of stability for (1).

Proof: According to the definition of nonlinearities ${ }^{2} \phi_{j}, j=$ $1, \ldots, p$, by applying Lemma $1 p$-times with adequate vectors $v, w$ and $v_{0}$, it follows that:

- First, in the case $\varphi=\phi_{1}$, relation (6) and definition (5) apply with

$$
T=T_{1} ; v=C x ; w=E_{11} x ; v_{0}=u_{1} .
$$

- Second, in the case $\varphi=\phi_{2}$, relation (6) and definition (5) apply with

$$
\begin{aligned}
& T=T_{2} ; v=\left(A_{1}+B_{1} C\right) x+B_{1} \phi_{1} ; \\
& w=E_{22} x+E_{21} \phi_{1} ; v_{0}=u_{2} .
\end{aligned}
$$

- ...

- Finally, in the case $\varphi=\phi_{p}$, relation (6) and definition (5) apply with

$$
\begin{aligned}
T= & T_{p} \\
v= & \left(A_{p-1}+B_{p-1}\left(A_{p-2}+B_{p-2}\right.\right. \\
& \left.\left.\quad \times\left(A_{p-3}+\cdots+B_{2}\left(A_{1}+B_{1} C\right)\right)\right)\right) x \\
& +B_{p-1} \phi_{p-1}+B_{p-1} B_{p-2} \phi_{p-2} \\
& +\cdots+B_{p-1} B_{p-2} \cdots B_{1} \phi_{1} \\
& +\cdots+E_{p 1} \phi_{1} ; v_{0}=u_{p} .
\end{aligned}
$$

Matrices $E_{11}, E_{22}, E_{21}, \ldots E_{p p}$, and $E_{p p-1}, \ldots, E_{p 1}$ are matrices of appropriate dimensions to be determined and matrices $T_{1}$, $T_{2}, \ldots, T_{p}$ are $p$ diagonal and positive-definite matrices.

Consider now $E_{j j}=Z_{j j} W^{-1}, j=1, \ldots, p$ and $E_{j l}=Y_{j l} S_{l}^{-1}$, $j=2, \ldots, p, l \neq j, j>l$. The satisfaction of relation (9) implies then

${ }^{1}$ The symbol $\star$ stands for symmetric blocks.

${ }^{2}$ For notational simplicity, we denote throughout the proof $\phi_{j}(x)$ by $\phi_{j}$. that the set $\mathcal{E}\left(W^{-1}, 1\right)$, with $P=W^{-1}$, is included in $S\left(u_{1}\right)$ [2]. We prove now, by induction, that relations (9) and (10) imply that set $\mathcal{E}\left(W^{-1}, 1\right)$ is included in $\cap_{j=1}^{p} S\left(u_{j}\right)$. First note that, from Lemma 1 , if $\mathcal{E}\left(W^{-1}, 1\right) \subset S\left(u_{1}\right)$, it follows that $\phi_{1}^{\prime} T_{1}\left(\phi_{1}+E_{11} x\right) \leq 0, \forall x \in$ $\mathcal{E}\left(W^{-1}, 1\right)$. Hence, provided that $\mathcal{E}\left(W^{-1}, 1\right) \subset S\left(u_{1}\right), \mathcal{E}\left(W^{-1}, 1\right)$ will be contained in $S\left(u_{1}\right) \cap S\left(u_{2}\right)$ if

$$
\begin{aligned}
& {\left[\begin{array}{c}
x \\
\phi_{1}
\end{array}\right]^{\prime}\left(\left[\begin{array}{ll}
P & 0 \\
0 & 0
\end{array}\right]-\frac{1}{u_{2(i)}^{2}}\left[\begin{array}{l}
\Gamma_{1(i)}^{\prime} \\
\Theta_{1(i)}^{\prime}
\end{array}\right]\left[\begin{array}{ll}
\Gamma_{1(i)} & \Theta_{1(i)}
\end{array}\right]\right)} \\
& \quad \times\left[\begin{array}{c}
x \\
\phi_{1}
\end{array}\right] \geq 0 \\
& \forall i=1, \ldots, m_{1} \\
& \forall x, \phi_{1} \text { such that } 2 \phi_{1}^{\prime} T_{1}\left(\phi_{1}+E_{11} x\right) \leq 0
\end{aligned}
$$

where $\Gamma_{1(i)}=A_{1(i)}-E_{22(i)}$ and $\Theta_{1(i)}=B_{1(i)}-E_{21(i)}$. Using the $S$-procedure and the Schur's complement, it follows that (11) is satisfied if

$\left[\begin{array}{ccc}P & \star & \star \\ T_{1} E_{11} & 2 T_{1} & \star \\ \mathrm{A}_{1(i)}-E_{22(i)} & B_{1(i)}-E_{21(i)} & u_{2(i)}^{2}\end{array}\right] \geq 0$

$\forall i=1, \ldots, m_{1}$.

Pre- and postmultiplying (12) by the matrix $\left[\begin{array}{ccc}W & \mathbf{0} & 0 \\ \star & S_{1} & \mathbf{0} \\ \star & \star & \mathbf{1}\end{array}\right]$, with $S_{1}=T_{1}^{-1}$ it follows that this inequality is equivalent to relation (10) for $j=2$.

Applying recursively the previous reasoning, provided that $\mathcal{E}\left(W^{-1}, 1\right) \subset \cap_{k=1}^{j-1} S\left(u_{k}\right)$, it follows that $\mathcal{E}\left(W^{-1}, 1\right)$ will be contained in $\cap_{k=1}^{j} S\left(u_{k}\right)$ if

$$
\begin{gathered}
{\left[\begin{array}{c}
x \\
\phi_{1} \\
\vdots \\
\phi_{j-1}
\end{array}\right]^{\prime}\left(\left[\begin{array}{cccc}
P & 0 & \ldots & 0 \\
\star & 0 & \ldots & 0 \\
\vdots & \vdots & \ddots & 0 \\
\star & \star & \ldots & \mathbf{0}
\end{array}\right]-\frac{1}{u_{j(i)}^{2}} \Omega^{\prime} \Omega\right)} \\
\forall i=1, \ldots, m_{j}
\end{gathered}
$$

$\forall x, \phi_{1}, \ldots, \phi_{j-1}$ such that

$$
\left\{\begin{array}{l}
2 \phi_{1}^{\prime} T_{1}\left(\phi_{1}+E_{11} x\right) \leq 0 \\
\vdots \\
2 \phi_{j-1}^{\prime} T_{j-1}\left(\phi_{j-1}+E_{j-1 j-1} x+E_{j-11} \phi_{1}\right. \\
\left.\quad+\ldots+E_{j-1 j-2} \phi_{j-2}\right) \leq 0
\end{array}\right.
$$

where $\Omega=\left[\begin{array}{llll}\Gamma_{j-1(i)} & \Theta_{1(i)} \ldots \Theta_{j-1(i)}\end{array}\right], \Gamma_{j-1(i)}=A_{j-1(i)}-E_{j j(i)}$, and $\Theta_{1(i)}=B_{j-1(i)} B_{j-2} \ldots B_{1}-E_{j 1(i)}, \ldots, \Theta_{j-1(i)}=B_{j-1(i)}-$ $E_{j j-1(i)}$, whence, using $S$-procedure, Schur's complement and considering $S_{j}=T_{j}^{-1}, j=2, \ldots, p$, it follows (10). Hence, $\forall x \in$

$$
\left[\begin{array}{cccccc}
W & Z_{11}^{\prime} & Z_{22}^{\prime} & \cdots & Z_{j-1 j-1}^{\prime} & W A_{j-1(i)}^{\prime}-Z_{j j(i)}^{\prime} \\
\star & 2 S_{1} & Y_{21}^{\prime} & \cdots & Y_{j-11}^{\prime} & S_{1} B_{1}^{\prime} \cdots B_{j-2}^{\prime} B_{j-1(i)}^{\prime}-Y_{j 1(i)}^{\prime} \\
\star & \star & 2 S_{2} & \cdots & Y_{j-12}^{\prime} & S_{2} B_{2}^{\prime} \cdots B_{j-2}^{\prime} B_{j-1(i)}^{\prime}-Y_{j 2(i)}^{\prime} \\
\vdots & \vdots & \vdots & \vdots & \vdots & \vdots \\
\star & \star & \star & \star & 2 S_{j-1} & S_{j-1} B_{j-1(i)}^{\prime}-Y_{j j-1(i)}^{\prime} \\
\star & \star & \star & \star & \star & u_{j(i)}^{2}
\end{array}\right] \geq 0, \quad i=1, \ldots, m_{j}, j=2, \ldots, p
$$


$\mathcal{E}\left(W^{-1}, 1\right)$, the satisfaction of relations (9) and (10) implies that set $\mathcal{E}\left(W^{-1}, 1\right)$ is included in $\cap_{j=1}^{p} S\left(u_{j}\right)$. Hence, the nonlinearities $\phi_{j}$, $j=1, \ldots, p$, associated to the appropriate $v$ and $w$ defined previously, satisfy sector conditions in the form of (6) for all $x \in \mathcal{E}\left(W^{-1}, 1\right)$.

Consider now the quadratic Lyapunov function $V(x)=x^{\prime} P x$, with $P=P^{\prime}>0$. The time-derivative of $V(x)$ along the trajectories of system (4) reads

$$
\begin{aligned}
\dot{V}=x^{\prime}\left(\mathrm{A}_{p}^{\prime} P\right. & \left.+P \mathrm{~A}_{p}\right) x+2 x^{\prime} P B_{p} \phi_{p} \\
& +2 x^{\prime} P B_{p} B_{p-1} \phi_{p-1}+\cdots+2 x^{\prime} P B_{p} B_{p-1} \cdots B_{1} \phi_{1} .
\end{aligned}
$$

Since (9) and (10) are satisfied, sector conditions (6) hold $\forall \phi_{j}, j=$ $1, \ldots, p, \forall x \in \mathcal{E}\left(W^{-1}, 1\right)$. Hence, $\forall x \in \mathcal{E}\left(W^{-1}, 1\right)$ it follows that

$\dot{V} \leq \dot{V}-2 \phi_{1}^{\prime} T_{1}\left(\phi_{1}+E_{11} x\right)-2 \phi_{2}^{\prime} T_{2}\left(\phi_{2}+E_{22} x+E_{21} \phi_{1}\right)-\cdots$ $-2 \phi_{p}^{\prime} T_{p}\left(\phi_{p}+E_{p p} x+E_{p p-1} \phi_{p-1}+E_{p p-2} \phi_{p-2}+\ldots+E_{p 1} \phi_{1}\right)$.

Considering $\xi=\left[\begin{array}{llll}x^{\prime} & \phi_{1}^{\prime} \phi_{2}^{\prime} \ldots \phi_{p}^{\prime}\end{array}\right]^{\prime}$, the previous inequality can be rewritten as $\dot{V} \leq \xi^{\prime} \mathcal{M} \xi$ with the equation shown at the bottom of the page.

By recalling that $W=P^{-1}, S_{j}=T_{j}^{-1}, j=1, \ldots, p$, and by pre- and postmultiplying the matrix aforementioned $\mathcal{M}$ defined by $\left[\begin{array}{ccccc}W & \mathbf{0} & \mathbf{0} & \mathbf{0} & \mathbf{0} \\ \star & S_{1} & \mathbf{0} & \mathbf{0} & \mathbf{0} \\ \star & \star & S_{2} & \mathbf{0} & \mathbf{0} \\ \vdots & \vdots & \vdots & \vdots & \vdots \\ \star & \star & \star & \star & S_{p}\end{array}\right]$, it follows that, if relation (8) is satisfied, one has $\dot{V}(x)<0, \forall x \in \mathcal{E}\left(W^{-1}, 1\right), x \neq 0$. Hence, one can conclude that $\mathcal{E}\left(W^{-1}, 1\right)$ is a contractive set along the trajectories of system (1) and, thus, it is a region where the asymptotic stability of system (1) is ensured, which concludes the proof.

\section{B. Global Asymptotic Stability Analysis}

Proposition 1 presents a local stability condition for the nested saturated system (1). On the other hand, in the case where $A_{p}$ is Hurwitz the global asymptotic stability of the system can be checked. In this case, following the results based on integral quadratic constraints (IQCs) stated in [4], [12] for systems presenting repeated saturations, ${ }^{3}$ different nondiagonal multipliers $T$ can be used as follows.

Lemma 2: The nonlinearity $\varphi(v)=\operatorname{sat}_{v_{0}}(v)-v$ satisfies the following inequality:

$$
\varphi(v)^{\prime} T(\varphi(v)+v) \leq 0
$$

${ }^{3}$ By "repeated" saturations the authors in that papers mean the case where $(\operatorname{sat}(v))_{(i)}=\operatorname{sat}\left(v_{(i)}\right)=\operatorname{sign}\left(\left(v_{(i)}\right) \min \left(v_{0(i)},\left|v_{(i)}\right|\right)\right.$ with $v_{0(i)}=\bar{u}, \forall i=1, \ldots, m$ appear in an additive way. It should be highlighted that these "repeated" saturations do not appear "nested" as in our case. for any vector $v \in \Re^{m}$ and any matrix $T \in \Re^{m \times m}$ such that

$$
T_{(i, i)} v_{0(i)} \geq \sum_{j=1, i \neq j}^{m} v_{0(j)}\left|T_{(i, j)}\right|, \quad i=1, \ldots, m
$$

Proof: Recalling that $\varphi(v)=\left[\varphi\left(v_{(1)}\right) \ldots \varphi\left(v_{(m)}\right)\right]^{\prime}$ it follows that

$$
\begin{aligned}
\varphi(v)^{\prime} T(\varphi(v)+v)=\sum_{i=1}^{m} \varphi\left(v_{(i)}\right)\left[T_{(i, i)}\left(\varphi\left(v_{(i)}\right)+v_{(i)}\right)\right. \\
\left.+\sum_{i \neq j, j=1}^{m} T_{(i, j)}\left(\varphi\left(v_{(j)}\right)+v_{(j)}\right)\right]
\end{aligned}
$$

Consider now the following three cases.

- Case 1: $\varphi\left(v_{(i)}\right)>0$ In this case, $\varphi\left(v_{(i)}\right)+$ $v_{(i)}=-v_{0(i)}$. Hence if (15) is satisfied one has $\left[T_{(i, i)}\left(\varphi\left(v_{(i)}\right)+v_{(i)}\right)+\sum_{i \neq j, j=1}^{m} T_{(i, j)}\left(\varphi\left(v_{(j)}\right)+\right.\right.$ $\left.\left.v_{(j)}\right)\right] \leq-v_{0(i)} T_{(i, i)}+\sum_{i \neq j, j=1}^{m} v_{0(j)}\left|T_{(i, j)}\right| \leq 0$ and, therefore $\varphi\left(v_{(i)}\right)\left[T_{(i, i)}\left(\varphi\left(v_{(i)}\right)+v_{(i)}\right)+\right.$ $\left.\sum_{i \neq j, j=1}^{m} T_{(i, j)}\left(\varphi\left(v_{(j)}\right)+v_{(j)}\right)\right] \leq 0$.

- Case 2: $\varphi\left(v_{(i)}\right)<0$ In this case, $\varphi\left(v_{(i)}\right)+$ $v_{(i)}=v_{0(i)}$. Hence if (15) is satisfied one has $\left[T_{(i, i)}\left(\varphi\left(v_{(i)}\right)+v_{(i)}\right)+\sum_{i \neq j, j=1}^{m} T_{(i, j)}\left(\varphi\left(v_{(j)}\right)+\right.\right.$ $\left.\left.v_{(j)}\right)\right] \geq v_{0(i)} T_{(i, i)}-\sum_{i \neq j, j=1}^{m} v_{0(j)}\left|T_{(i, j)}\right| \geq 0$ and, therefore $\varphi\left(v_{(i)}\right)\left[T_{(i, i)}\left(\varphi\left(v_{(i)}\right)+v_{(i)}\right)+\right.$ $\left.\sum_{i \neq j, j=1}^{m} T_{(i, j)}\left(\varphi\left(v_{(j)}\right)+v_{(j)}\right)\right] \leq 0$.

- Case 3: $\varphi\left(v_{(i)}\right)=0$ One has $\varphi\left(v_{(i)}\right)\left[T_{(i, i)}\left(\varphi\left(v_{(i)}\right)+v_{(i)}\right)+\right.$ $\left.\sum_{i \neq j, j=1}^{m} T_{(i, j)}\left(\varphi\left(v_{(j)}\right)+v_{(j)}\right)\right]=0$.

Thus, from the three previous cases and from (16), we can conclude that $\varphi(v)^{\prime} T(\varphi(v)+v) \leq 0$.

Hence, by using Lemma 2, a condition for the global asymptotic stability can be stated.

Proposition 2: If there exist a matrix $P=P^{\prime}>0$ and matrices $T_{k}=T_{k}^{\prime}>0, k=1, \ldots, p$, of appropriate dimensions, satisfying (17) and (18), as shown at the bottom of the next page. Then, the origin of system (1) is globally asymptotically stable.

Proof: It follows the same steps of Proposition 1, considering $w=v$. In this case, we should consider $E_{11}=C$, $E_{22}=A_{1}+B_{1} C, \ldots, E_{p p}=\left(A_{p-1}+B_{p-1}\left(A_{p-2}+B_{p-2}\left(A_{p-3}+\right.\right.\right.$ $\left.\left.\left.\cdots+B_{2}\left(A_{1}+B_{1} C\right)\right)\right)\right)=A_{p-1}, E_{21}=B_{1}, E_{p p-1}=$ $B_{p-1}, \ldots, E_{p 1}=B_{p-1} B_{p-2} \ldots B_{2} B_{1}$. It follows that the sector conditions (14), applied $p$-times to the nonlinearities defined in (2) are satisfied $\forall x \in \Re^{n}$.

Remark 2: If the control bounds are normalized to $v_{0(i)}=1, i=$ $1, \ldots, m$, condition (15) reads $T_{(i, i)} \geq \sum_{j=1, j \neq i}^{m}\left|T_{(i, j)}\right|$, which corresponds to the one given in [4] from an IQC approach. Furthermore, in order to implement (18) in an LMI framework we can consider

$$
\mathcal{M}=\left[\begin{array}{ccccc}
\mathrm{A}_{p}^{\prime} P+P \mathrm{~A}_{p} & \star & \star & \cdots & \star \\
B_{1}^{\prime} \cdots B_{p-1}^{\prime} B_{p}^{\prime} P-T_{1} E_{11} & -2 T_{1} & \star & \cdots & \star \\
B_{2}^{\prime} \cdots B_{p-1}^{\prime} B_{p}^{\prime} P-T_{2} E_{22} & -T_{2} E_{21} & -2 T_{2} & \cdots & \star \\
B_{3}^{\prime} \cdots B_{p-1}^{\prime} B_{p}^{\prime} P-T_{3} E_{33} & -T_{3} E_{31} & -T_{3} E_{32} & \cdots & \star \\
\vdots & \vdots & \vdots & \cdots & \star \\
B_{p}^{\prime} P-T_{p} E_{p p} & -T_{p} E_{p 1} & -T_{p} E_{p 2} & \cdots & -2 T_{p}
\end{array}\right]
$$


$T_{k(i, j)} \triangleq T_{k(i, j)}^{+}+T_{k(i, j)}^{-}$, for $i \neq j$, with $T_{k(j, i)}^{+}=T_{k(i, j)}^{+} \geq 0$ and $T_{k(i, j)}^{-}=T_{k(j, i)}^{-} \leq 0$ and the following constraints [4]

$\begin{aligned} u_{k(i)} T_{k(i, i)} \geq \sum_{j=1, j \neq i}^{m} u_{k(j)}\left(T_{k(i, j)}^{+}-T_{k(i, j)}^{-}\right), & \\ & k=1, \ldots, p, i=1, \ldots, m_{k} .\end{aligned}$

\section{Theoretical Synthesis Conditions}

Let us define the following matrices:

$$
\begin{aligned}
\mathbb{G}_{j-1}=G_{j-1}+B_{j-1} & \left(G_{j-2}+B_{j-2}\left(G_{j-3}+\cdots\right.\right. \\
+ & \left.\left.B_{2}\left(G_{1}+B_{1} G_{0}\right)\right)\right), \quad j=2, \ldots, p
\end{aligned}
$$

The following proposition provides theoretical sufficient conditions to address Problem 2.

Proposition 3: If there exist a symmetric positive-definite matrix $W$, matrices $Z_{j j}, j=1, \ldots, p, Y_{j l}, j=2, \ldots, p, l=1, \ldots, p-1$, $j \neq l, j>l, G_{k}, k=0, \ldots, p-1$, and diagonal positive matrices $S_{j}$, $j=1, \ldots, p$, of appropriate dimensions satisfying

$$
\begin{gathered}
{\left[\begin{array}{ccccc}
L+L^{\prime} & \star & \star & \cdots & \star \\
S_{1} B_{1}^{\prime} \ldots B_{p-1}^{\prime} B_{p}^{\prime}-Z_{11} & -2 S_{1} & \star & \cdots & \star \\
S_{2} B_{2}^{\prime} \ldots B_{p-1}^{\prime} B_{p}^{\prime}-Z_{22} & -Y_{21} & -2 S_{2} & \cdots & \star \\
\vdots & \vdots & \vdots & \cdots & \star \\
S_{p} B_{p}^{\prime}-Z_{p p} & -Y_{p 1} & -Y_{p 2} & \cdots & -2 S_{p}
\end{array}\right]<0} \\
{\left[\begin{array}{ccc}
W & G_{0(i)}^{\prime}-Z_{11(i)}^{\prime} \\
\star & u_{1(i)}^{2}
\end{array}\right] \geq 0, i=1, \ldots, m_{1}}
\end{gathered}
$$

and (22), as shown at the bottom of the page, holds, with

$$
L=A_{p} W+B_{p} G_{p-1}+\ldots+B_{p} B_{p-1} \ldots B_{2}\left(G_{1}+B_{1} G_{0}\right)
$$

Then, the gains $A_{j-1}=G_{j-1} W^{-1}, j=2, \ldots, p$ and $C=G_{0} W^{-1}$, are such that the asymptotic stability of (1) is ensured for all initial conditions belonging to the set $\mathcal{E}\left(W^{-1}, 1\right)=\left\{x \in \Re^{n} ; x^{\prime} W^{-1} x \leq 1\right\}$.

Proof: It follows the same lines as those of Proposition 1 by using the change of variables $A_{j-1}=G_{j-1} W^{-1}, j=2, \ldots, p$ and $C=$ $G_{0} W^{-1}$

The following result allows to address the global stabilizing gains design.

Corollary 1: If there exist a symmetric positive definite matrix $W$, $G_{k}, k=0, \ldots, p-1$, and diagonal positive matrices $S_{j}, j=1, \ldots, p$, of appropriate dimensions satisfying (24), as shown at the bottom of the page, where $\mathbb{G}_{p-1}$ and $L$ are defined in (19) and (23), respectively, then (1) with $A_{j-1}=G_{j-1} W^{-1}, j=2, \ldots, p$ and $C=G_{0} W^{-1}$, is globally asymptotically stable.

Remark 3: Differently from the analysis case, the multipliers $T_{j}$, $j=1, \ldots, p$ cannot be generalized to satisfy (15). Indeed, instead of matrices $T_{j}$, condition (24) is formulated using their inverses $S_{j}$. Hence, considering a generic $m_{j}>2$ it is not possible to find a particular structure for $S_{j}$ which would ensure that $T_{j}$ would satisfy (18). However, note that for $m_{j}=2$ this can be ensured if the following additional constraints are considered:

$$
\begin{aligned}
& u_{j(1)} S_{j(1,1)}>u_{j(2)}\left|S_{j(1,2)}\right| \\
& u_{j(2)} S_{j(2,2)}>u_{j(1)}\left|S_{j(1,2)}\right|=u_{j(1)}\left|S_{j(2,1)}\right|
\end{aligned}
$$

Only in this particular case it is therefore possible to find matrices $T_{j}=$ $S_{j}^{-1}, j=1, \ldots, p$, satisfying (17) and (18).

$$
\begin{aligned}
& {\left[\begin{array}{ccccc}
\mathbb{A}_{p}^{\prime} P+P A_{p} & P B_{p} B_{p-1} \ldots B_{1}-C^{\prime} T_{1} & P B_{p} B_{p-1} \ldots B_{2}-\left(A_{1}+B_{1} C\right)^{\prime} T_{2} & \ldots & P B_{p}-\mathbb{A}_{p-1}^{\prime} T_{p} \\
\star & -2 T_{1} & -B_{1}^{\prime} T_{2} & \ldots & -\left(B_{p-1} \ldots B_{1}\right)^{\prime} T_{p} \\
\star & \star & -2 T_{2} & \ldots & -\left(B_{p-1} \ldots B_{2}\right)^{\prime} T_{p} \\
\vdots & \vdots & \vdots & \vdots & \vdots \\
\star & \star & \star & \star & -2 T_{p}
\end{array}\right]<0} \\
& u_{k(i)} T_{k(i, i)} \geq \sum_{j=1, j \neq i}^{m_{k}} u_{k(j)}\left|T_{k(i, j)}\right|, \quad k=1, \ldots, p, i=1, \ldots, m_{k}
\end{aligned}
$$

$$
\left[\begin{array}{cccccc}
W & Z_{11}^{\prime} & Z_{22}^{\prime} & \ldots & Z_{j-1 j-1}^{\prime} & \mathbb{G}_{j-1(i)}^{\prime}-Z_{j j(i)}^{\prime} \\
\star & 2 S_{1} & Y_{21}^{\prime} & \ldots & Y_{j-11}^{\prime} & S_{1} B_{1}^{\prime} \ldots B_{j-2}^{\prime} B_{j-1(i)}^{\prime}-Y_{j 1(i)}^{\prime} \\
\star & \star & 2 S_{2} & \ldots & Y_{j-12}^{\prime} & S_{2} B_{2}^{\prime} \ldots B_{j-2}^{\prime} B_{j-1(i)}^{\prime}-Y_{j 2(i)}^{\prime} \\
\vdots & \vdots & \vdots & \vdots & \vdots & \vdots \\
\star & \star & \star & \star & 2 S_{j-1} & S_{j-1} B_{j-1(i)}^{\prime}-Y_{j j-1(i)}^{\prime} \\
\star & \star & \star & \star & \star & u_{j(i)}^{2}
\end{array}\right] \geq 0 \quad i=1, \ldots, m_{j}, j=2, \ldots, p
$$

$$
\left[\begin{array}{ccccc}
L+L^{\prime} & \star & \star & \cdots & \star \\
S_{1} B_{1}^{\prime} \ldots B_{p-1}^{\prime} B_{p}^{\prime}-G_{0} & -2 S_{1} & \star & \cdots & \star \\
S_{2} B_{2}^{\prime} \ldots B_{p-1}^{\prime} B_{p}^{\prime}-\left(G_{1}+B_{1} G_{0}\right) & -B_{1} S_{1} & -2 S_{2} & \cdots & \star \\
\vdots & \vdots & \vdots & \ldots & \star \\
S_{p} B_{p}^{\prime}-G_{p-1} & -B_{p-1} \ldots B_{1} S_{1} & -B_{p-1} \ldots B_{2} S_{2} & \cdots & -2 S_{p}
\end{array}\right]<0
$$




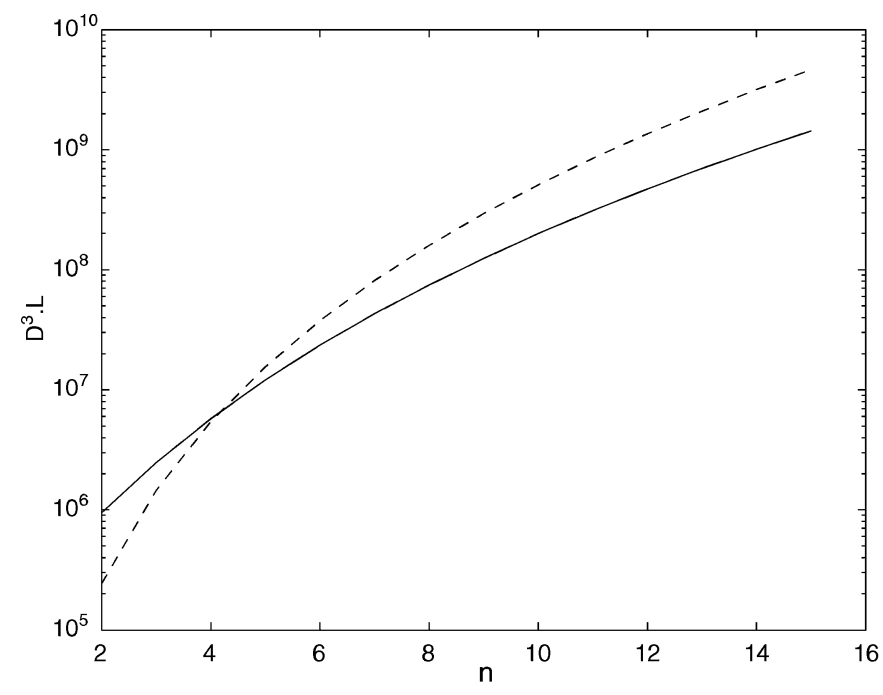

(a)

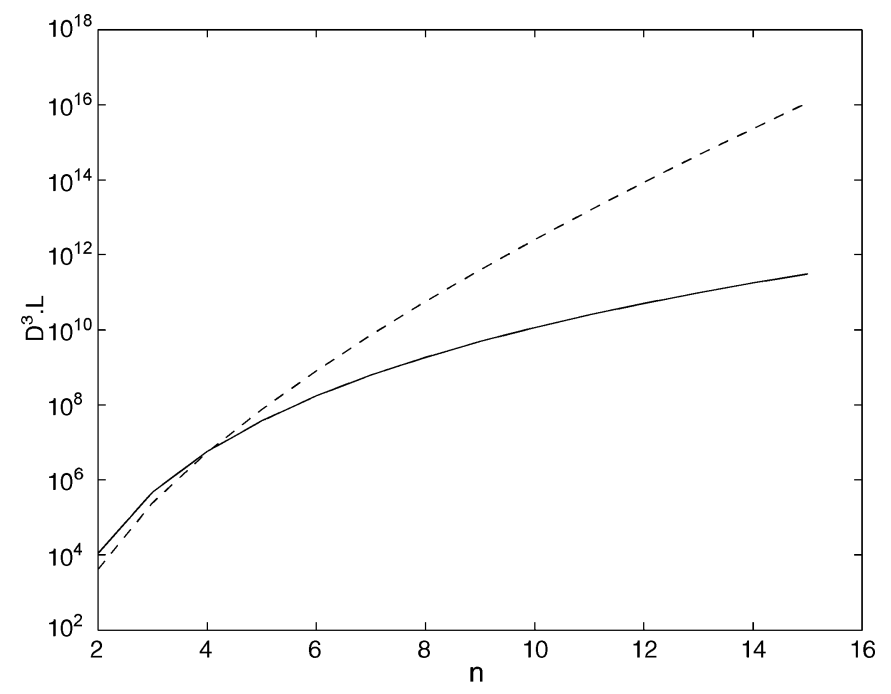

(b)

Fig. 1. $\mathcal{D}^{3} \mathcal{L}$ for $p=2$ in function of $n$ from Proposition 1 (solid line) and from [1, Th. 2.1] (dashed line): For (a) $m=3$ and for (b) $m=n-1$.

The same problem appears in the local stability and stabilization contexts. Note that, in these cases, the conditions are formulated in terms of matrices $S_{j}$, instead of the direct multipliers $T_{j}$.

\section{OPTIMIZATION ISSUE}

From the result stated in Propositions 1 and 3, we are interested in one of the following cases.

1) A set of initial states, $\Xi_{0} \subset \Re^{n}$, for which asymptotic stability must be ensured, is given.

2) We aim to maximize the estimate of the basin of attraction associated to (1). In other words, we want to compute the set $\mathcal{E}\left(W^{-1}, 1\right)$ as large as possible considering some size criterion.

Both cases can be addressed if we consider a set $\Xi_{0}$ with a given shape and a scaling factor $\beta$. For example, let $\Xi_{0}$ be defined as a polyhedral set described by its vertices: $\Xi_{0}=\operatorname{Co}\left\{v_{r} ; r=1, \ldots, n_{r}, v_{r} \in\right.$ $\left.\Re^{n}\right\}$. We want then to satisfy $\beta \Xi_{0} \subset \mathcal{E}\left(W^{-1}, 1\right)$. In case 1$)$, this problem reduces to a feasibility problem with $\beta=1$. In case 2 ), the goal consists in maximizing $\beta$, which corresponds to define through $\Xi_{0}$ the directions in which we want to maximize $\mathcal{E}\left(W^{-1}, 1\right)$.

The problem of maximizing $\beta$ can be accomplished by solving the following convex optimization problem:

$\min \mu$

subject to relations (8),(9),(10) (in the analysis context)

subject to relations (20),(21),(22)

(in the synthesis context)

$$
\left[\begin{array}{ll}
\mu & v_{r}^{\prime} \\
v_{r} & W
\end{array}\right] \geq 0, \quad r=1, \ldots, n_{r} .
$$

Considering $\beta=1 / \sqrt{\mu}$, the minimization of $\mu$ implies the maximization of $\beta$. Note that other criteria associated to the size of $\mathcal{E}\left(W^{-1}, 1\right)$ (e.g., the volume or the size of the minor axis) can be adopted in order to maximize the stability region.

Remark 4: In a practical problem, the designer can also be requested to specify (or to choose) the actuators and/or sensors that better fit the design objectives for the system, under constraints on cost, size, energy consummation, mass, etc. In particular, the specification of the actuator or sensor can be done by its saturation amplitude bound $u_{j}$. The idea is therefore to consider $u_{j}, j=1, \ldots, p$ also as decision variables. This can be accomplished in the same LMI framework by replacing $u_{j(i)}^{2}$ by $\eta_{j(i)}, i=1, \ldots, m_{j}, j=1, \ldots, p$, in (21) and (22). In this case, considering a given set $\Xi_{0}$ for which the stability should be ensured, an interesting optimization problem could be to consider a linear cost criterion on $\eta_{j(i)}, i=1, \ldots, m_{j}, j=1, \ldots, p$.

\section{A. Computational Burden Analysis}

The conditions of Proposition 1 are under LMI form in the decision variables. This fact is due to the use of model (4) with Lemma 1. The use of polytopic model as that one described in [1] also leads to LMI conditions. Let us now compare the number of variables and the number of lines in the LMIs to be solved considering our Proposition 1 and the LMIs considered in [1, Th. 2.1].

Consider $m_{1}=\cdots=m_{p}=m$, where $m$ is a fixed integer and assume that all matrices $B_{j}$, for $1 \leq j \leq p-1$ are diagonal such that the results of [1] apply.

In Proposition 1, we have to consider LMI conditions with

- $n+m p+(n+1) m+\sum_{j=2}^{p} m(n+m(j-1)+1)=n+m[p+$ $p(n+1)+m(p(p-1) / 2)]$ lines;

- $(n(n+1) / 2)+p m n+(p(p-1) / 2) m^{2}+m p$ decision variables (for $W, Z_{j j}, Y_{j l}$ and $S_{j}$, respectively).

On the other hand, in [1, Th. 2.1], to solve the analysis problem we need to consider LMI conditions with

- $n(p+1)^{m}+(n+1) m p$ lines;

- $m n p+(n(n+1) / 2)$ decision variables.

For small values of $n, m$, and $p$, the number of variables and lines do not slightly differ, and LMI problems to be considered in Proposition 1 and in [1, Th. 2.1] are numerically similar. However, when the complexity analysis of the numerical method is addressed, the numbers of lines and of variables of the considered LMIs play a crucial role. Indeed, the computation gets more complex when these numbers become larger: see, for example the complexity analysis of interior-point method [6], or the complexity analysis for a primal method [5, Th. 5.1] or for a dual method [5, Th, 5.2]. LMI conditions can be solved in polynomial time for instance by specialized algorithms as in [6], with complexity proportional to $\mathcal{D}^{3} \mathcal{L}$ (where $\mathcal{D}$ is the number of decision variables and $\mathcal{L}$ is the number of lines). Of course, other LMI solvers may perform differently. When we study this quantity (like in Fig. 1), one can observe that for high dimensions, the numerical complexity associated to our conditions increases slower than that one associated 
to [1, Th.2.1], due in particular to the fact that the number of lines in [1, Th.2.1] depends on a term $(p+1)^{m}$.

Note that the same type of computational burden analysis as developed before can be done for the synthesis case.

\section{ILLUSTRATIVE EXAMPLES}

Example 1: Consider the numerical example borrowed from [1], in which system (1) with $p=2, m_{2}=m_{1}=1$ and $n=2$, is described by the following data:

$$
A_{2}=\left[\begin{array}{cc}
0 & -0.5 \\
1 & 1.5
\end{array}\right] \quad B_{2}=\left[\begin{array}{c}
0 \\
-1
\end{array}\right] \quad B_{1}=0.1 \quad u_{2}=u_{1}=1 .
$$

By considering the same conditions as in Example 2.2, [1, Case 4], and by applying Proposition 3 one obtains

$$
\begin{aligned}
A_{1} & =\left[\begin{array}{ll}
0.4878 & 203.4825
\end{array}\right] C=\left[\begin{array}{ll}
-0.0057 & 0.0284
\end{array}\right] \\
P & =\left[\begin{array}{cc}
2.2809 & -0.0010 \\
-0.0010 & 2.6232
\end{array}\right] .
\end{aligned}
$$

The obtained ellipsoid $\mathcal{E}\left(W^{-1}, 1\right)$ is the same than that one provided in [1].

Example 2: Consider the longitudinal dynamics of the F-8 aircraft borrowed from [19]. System (1), in the case $p=3, m_{3}=m_{2}=2$, $m_{1}=1$ and $n=4$, is described by the following data:

$$
\begin{aligned}
& A_{3}= {\left[\begin{array}{cccc}
-0.8 & -0.006 & -12 & 0 \\
0 & -0.014 & -16.64 & -32.2 \\
1 & -0.0001 & -1.5 & 0 \\
1 & 0 & 0 & 0
\end{array}\right] } \\
& B_{3}=\left[\begin{array}{cc}
-19 & -3 \\
-0.66 & -0.5 \\
-0.16 & -0.5 \\
0 & 0
\end{array}\right] \\
& B_{2}=\left[\begin{array}{cc}
1 & -1 \\
0 & 1
\end{array}\right] \quad B_{1}=\left[\begin{array}{l}
0.1 \\
0.1
\end{array}\right] u_{3}=u_{2}=\left[\begin{array}{l}
15 \\
15
\end{array}\right] \quad u_{1}=15
\end{aligned}
$$

Let us consider $\Xi_{0}$ as the unit ball in $\Re^{4}$ for the usual norm $l^{\infty}$. Note that, since $m_{3}, m_{2} \neq m_{1}$, the approach of [1] does not seem applicable. By applying Proposition 3 and by considering an additional pole placement constraint [8] such as $R_{e}\left(\lambda_{i}\left(A_{3}+B_{3}\left(A_{2}+B_{2}\left(A_{1}+\right.\right.\right.\right.$ $\left.\left.\left.B_{1} C\right)\right)\right) \leq-0.5$, one obtains

$$
\begin{aligned}
A_{2} & =\left[\begin{array}{cccc}
0.0185 & -0.0018 & -0.0937 & 0.1152 \\
-0.0185 & 0.0018 & 0.0937 & -0.1152
\end{array}\right] \\
A_{1} & =\left[\begin{array}{llll}
0.0185 & -0.0018 & -0.0937 & 0.1152 \\
0.0180 & -0.0017 & -0.0917 & 0.1125
\end{array}\right] \\
C & =\left[\begin{array}{llll}
0.0086 & -0.0008 & -0.0442 & 0.0542
\end{array}\right] \\
\beta & =435.8886
\end{aligned}
$$

with $\operatorname{eig}\left(A_{3}+B_{3}\left(A_{2}+B_{2}\left(A_{1}+B_{1} C\right)\right)\right)=\{-1.1596 \pm$ $j 3.4520 ;-0.5002 \pm j 0.2904\}$.

Example 3: Consider system (1), in the case $p=2, m_{1}=m_{2}=1$ and $n=2$, described by the following data:

$$
A_{2}=\left[\begin{array}{cc}
0 & 1 \\
-1 & -2
\end{array}\right] \quad B_{2}=\left[\begin{array}{c}
0 \\
-1
\end{array}\right] \quad B_{1}=1 u_{1}=u_{2}=1 .
$$

By applying Corollary 1, it follows that this systems can be globally asymptotically stabilized with

$$
A_{1}=\left[\begin{array}{ll}
-0.0264 & -0.3423
\end{array}\right] \quad C=\left[\begin{array}{ll}
-0.1422 & -0.5441
\end{array}\right] .
$$

\section{CONCLUSION}

An LMI-based methodology to address the problems of stability and stabilization of systems presenting nested saturations has been proposed. This methodology is based on the modelling of the system as a linear system with nested deadzone nonlinearities and on the use of a generalized sector condition. In particular, differently from [1], the proposed approach allows to consider more general nested saturation structures and to address the global stability and global stabilization problems. On the other hand, in the case where the global asymptotic stability of the system can be checked, the results concerning the use of more generic multipliers, stated in [4], and [12] for systems presenting repeated saturations, are adapted to the present case of nested saturations. Some examples borrowed from the literature highlighted the application of the results.

The present study opens several perspectives for future works. In particular, given a general linear system $\dot{x}=A x$, the complete synthesis problem i.e. the problem of the design of all gains (namely all matrices $A_{j}$ and $B_{j}$ in (1)) is still open. Existence of such a global stabilizing saturation control is proved in [17] for multiple integrators and is used in [13] for feedforward systems with delayed inputs. In this note, only a part of this set of gains (namely, the matrices $A_{j}=G_{j} W^{-1}$, $j=1, \ldots, p$ and $C=G_{0} W^{-1}$ in Corollary 1 ) have been designed. Note that it is also possible to design the bound of the saturation function $u_{j}$ since the LMI condition stated in Corollary 1 is linear in $u_{j(i)}^{2}$. However, the design of the other gains (i.e., the matrices $B_{j}$ ) is a more challenging problem and is currently under investigation. Other perspectives regard the use of such nested saturations for the global stabilization of cascade systems with linear part (see [15], [17], and [18]) and the study of different nonlinearities than the nested saturation (see, e.g., the hysteresis in [11]).

\section{REFERENCES}

[1] A. Bateman and Z. Lin, "An analysis and design method for linear systems under nested saturation,” Syst. Control Lett., vol. 48, pp. 41-52, 2003.

[2] S. Boyd, L. El Ghaoui, E. Feron, and V. Balakrishnan, Linear Matrix Inequalities in Systems and Control Theory. Philadelphia, PA: SIAM.

[3] P. J. Campo and M. Morari, "Robust control of processes subject to saturations nonlinearities," Comput. Chem. Eng., vol. 14, no. 4-5, pp. 343-358, 1990.

[4] F. J. D'Amato, M. A. Rotea, A. V. Megretski, and U. T. Jönsson, "New results for analysis of systems with repeated nonlinearities," $\mathrm{Au}$ tomatica, vol. 37, no. 5, pp. 739-747, 2001.

[5] E. de Klerk, Aspects of Semidefinite Programming. Interior Point Algorithms and Selected Applications. Dordrecht, The Netherlands: Kluwer, 2002.

[6] P. Gahinet, A. Nemirovski, A. J. Laub, and M. Chilali, LMI Control Toolbox User's Guide. Natick, MA: The MathWorks, Inc., 1995.

[7] J. M. Gomes da Silva, Jr. and S. Tarbouriech, "Anti-windup design with guaranteed region of stability: An LMI-based approach," IEEE Trans. Autom. Control, vol. 50, no. 1, pp. 106-111, Jan. 2005.

[8] J. M. Gomes da Silva, Jr., S. Tarbouriech, and G. Garcia, "Local stabilization of linear systems under amplitude and rate saturating actuators," IEEE Trans. Autom. Control, vol. 48, no. 5, pp. 842-847, May 2003.

[9] H. Hindi and S. Boyd, "Analysis of linear systems with saturation using convex optimization," in Proc. IEEE Conf. Decision and Control, San Diego, CA, Dec. 1998, pp. 903-908.

[10] V. Kapila and K. M. Grigoriadis, Eds., Actuator Saturation Control. New York: Marcel Dekker, 2002.

[11] H. K. Khalil, Nonlinear Systems, 2nd ed. London, U.K.: PrenticeHall, 1996

[12] V. V. Kulkarni and M. G. Safonov, "All multipliers for repeated monotone nonlinearities," in Proc. Amer. Control Conf., Arlington, VA, Jun. 2001.

[13] F. Mazenc, S. Mondié, and R. Francisco, "Global asymptotic stabilization of feedforward systems with delay in the input," IEEE Trans. Autom. Control, vol. 49, no. 5, pp. 844-850, May 2004. 
[14] T. Nguyen and F. Jabbari, "Output feedback controllers for disturbance attenuation with actuator amplitude and rate saturation," Automatica, vol. 36, pp. 1339-1346, 2000.

[15] R. Sepulchre, M. Jankovic, and P. Kokotovic, Constructive Nonlinear Control. London, U.K.: Springer-Verlag, 1997.

[16] S. Tarbouriech, I. Queinnec, and G. Garcia, "Stability region enlargement through anti-windup strategy for linear systems with dynamics restricted actuator," Int. J. Syst. Sci., vol. 37, no. 2, pp. 79-90, 2006.

[17] A. R. Teel, "Global stabilization and restricted tracking for multiple integrators with bounded controls," Syst. Control Lett., vol. 18, pp. 165-171, 1992.

[18] A. R. Teel, "Feedback stabilization: Nonlinear solutions to inherently nonlinear problems," Ph.D. dissertation, Univ. California, Berkeley, CA, 1992.

[19] F. Wu, K. M. Grigoriadis, and A. Packard, "Anti-windup controller analysis via linear parameter-varying control design methods," in Proc. Amer. Control Conf., Philadelphia, PA, Jun. 1998, pp. 343-347.

\section{A Numerical Analysis of the Nash Strategy for Weakly Coupled Large-Scale Systems}

\author{
Hiroaki Mukaidani
}

\begin{abstract}
This note discusses the feedback Nash equilibrium of linear quadratic $\boldsymbol{N}$-player Nash games for infinite-horizon large-scale interconnected systems. The asymptotic structure along with the uniqueness and positive semidefiniteness of the solutions of the cross-coupled algebraic Riccati equations (CAREs) is newly established via the Newton-Kantorovich theorem. The main contribution of this study is the proposal of a new algorithm for solving the CAREs. In order to improve the convergence rate of the algorithm, Newton's method is combined with a new decoupling algorithm; it is shown that the proposed algorithm attains quadratic convergence. Moreover, it is shown for the first time that solutions to the CAREs can be obtained by solving the independent algebraic Lyapunov equation (ALE) by using the reduced-order calculation.
\end{abstract}

Index Terms-Cross-coupled algebraic Riccati equations (CARE), fixedpoint algorithm, Nash games, Newton's method, weakly coupled large-scale systems.

\section{INTRODUCTION}

The stability analysis and control of large-scale systems has been extensively investigated (see, e.g., [1]). For example, these control problems can be illustrated by multiarea power systems [2], [3]. The control problems of large-scale interconnected systems is parameterized by a small weak coupling parameter $\varepsilon$. This has been extensively studied in [2], [3], [5].

The linear quadratic Nash games and their applications have been widely investigated in many literatures (see, e.g., [9], [24] and the references therein). In particular, the definition and standard results given in [24] will be used for reference. There exist two different types of Nash equilibria: a) open-loop equilibria and b) closed-loop no-memory and feedback equilibria. The existence of open-loop Nash equilibria has been studied in [16], [19], and [20] for both continuous and discrete

Manuscript received February 14, 2005; revised December 12, 2005 and April 21, 2006. Recommended by Associate Editor F. Bullo. This work was supported in part by the Research Foundation for the Electrotechnology of Chubu (REFEC).

The author is with the Graduate School of Education, Hiroshima University, Higashi-Hiroshima 739-8524, Japan (e-mail: mukaida@ hiroshima-u.ac.jp).

Digital Object Identifier 10.1109/TAC.2006.878744 time systems. Further, in the case of memoryless perfect-state information structure, the asymptotic analysis of the linear feedback Nash equilibria has also been investigated [21]-[23]. These researches have focused on the analysis of the existence of Nash equilibria. Although a numerical algorithm for solving the feedback Nash algebraic Riccati equations has been presented, only a scalar feedback has been considered [23].

It is well known that in order to obtain Nash strategies, the crosscoupled algebraic Riccati equations (CARE) must be solved. In [10], a Newton-type algorithm for solving the CARE has been applied. A variant of the classical eigenvector approach for solving the CARE of the open-loop Nash games has also been studied in [16]. However, the computing workspace for these techniques requires very large matrix dimensions. Therefore, the reduction in the matrix dimensions poses a crucial problem because the weakly coupled systems include numerous subsystems. In contrast, in [4], the recursive algorithms have been developed for solving the CARE of the weakly coupled systems. Recently, an algorithm that is based on the Lyapunov iterations for solving the CARE has been introduced [6], [7]. Although such algorithms can be computed by using the dimension of each subsystem, the convergence rate is given by the linear convergence. Moreover, there is no proof of whether the abovementioned algorithms fail to converge in the case of strongly coupled systems.

This note investigates the feedback Nash equilibrium of linear quadratic $N$-player Nash games for infinite-horizon large-scale interconnected systems by using Newton's method. It should be noted that this study considers the linear feedback strategy [18] with memoryless perfect-state information structure [21]-[24]. This note is an extension of [8] in the sense that the convergence criteria for $\varepsilon$ is derived for the first time. Such a condition is derived by applying the Newton-Kantorovich theorem. The Newton-Kantorovich theorem plays an important role in showing that the uniqueness and positive semidefiniteness of the convergence solutions are guaranteed in the neighborhood of the initial conditions. Moreover, the asymptotic structure of the solutions of the CARE is established without the implicit function theorem. This note also proposes a new decoupling algorithm for computing Newton's iterations as another important feature. As a result, a reduction in the computation is attained by using the existing fixed-point algorithm [6], [7].

Notation: The notations used in this note are fairly standard. block diag denotes the block diagonal matrix. The superscript $T$ denotes the matrix transpose. $I_{n}$ denotes the $n \times n$ identity matrix. $\|\cdot\|$ denotes the Euclidean norm for a matrix. vec $M$ denotes the column vector of the matrix $M$ [15]. det $M$ denotes the determinant of the matrix $M . \otimes$ denotes the Kronecker product. $\delta_{i j}$ denotes the Kronecker delta. Re $\lambda M$ denotes the real part of the eigenvalue of the matrix $M$.

\section{Problem Formulation}

Consider weakly coupled large-scale linear systems with $N$ players

$$
\begin{aligned}
\dot{x}_{i}(t)= & A_{i i} x_{i}(t)+B_{i i} u_{i}(t)+\varepsilon \sum_{j=1, j \neq i}^{N} A_{i j} x_{j}(t) \\
& +\varepsilon \sum_{j=1, j \neq i}^{N} B_{i j} u_{j}(t) \quad x_{i}(0)=x_{i}^{0}, \quad i=1,2, \ldots, N
\end{aligned}
$$

where $x_{i} \in \mathbf{R}^{n_{i}}, i=1,2, \ldots, N$ represent the $i$ th state vectors. $u_{i} \in \mathbf{R}^{m_{i}}, i=1,2, \ldots, N$ represent the $i$ th control inputs. $\varepsilon$ denotes a small weak coupling parameter that connects the other subsystems. Each player attempts to minimize its cost performance subject to (1) by exploiting the available information in order to take the correct decision 